\title{
PECULIARITIES OF TECHNOLOGY OF MANUFACTURE AND APPLICATION OF FLUX-CORED STRIPS FOR SURFACING
}

\author{
A.P. VORONCHUK, A.P. ZHUDRA, V.O. KOCHURA, A.V. PETROV and V.V. FEDOSENKO \\ E.O. Paton Electric Welding Institute, NASU \\ 11 Bozhenko Str., 03680, Kiev, Ukraine. E-mail: office@paton.kiev.ua
}

\begin{abstract}
The paper deals with the problems of homogeneity of flux-cored strip charge at its mixing in double-cone mixers. Optimum mixing time for mixers of different volume was determined experimentally. Equipment for manufacturing flux-cored strips was optimized and a new design of withdrawal-roll set using pressure sensors was developed, that allows determination and adjustment of press-down force of withdrawal rolls of the mill. The paper gives the results of studying the influence of the degree of charge compaction on welding-technological properties of flux-cored strips. It is found that increase degree of compaction leads to a noticeable increase of the coefficient of electrode material melting. Insufficient or excessive degree of compaction adversely affects the welding-technological properties of flux-cored strips - spattering is increased and process stability deteriorates. 4 Ref., 1 Table, 7 Figures.
\end{abstract}

Keywords: surfacing, flux-cored strip, charge, mixer, mixing, homogeneity, complex-alloyed powders, degree of compaction, spattering

Flux-cored strips are widely applied in surfacing large-sized parts in metallurgical, mining, power and other industries. This is, primarily, related to high efficiency of surfacing with flux-cored strip reaching up to $30 \mathrm{~kg}$ of deposited metal per hour at application of one strip. Flux-cored strip is simple to manufacture, and its design features allow obtaining the filling coefficient of 60 to $70 \%$, that enables an essential increase of the degree of deposited metal alloying [1].

Technology of flux-cored strip manufacturing has several features, associated with preparation of charge materials, as well as process of strip forming, compacting and rolling.

The paper deals with two problems that may have an essential influence on flux-cored strip quality - this is mixing of charge materials and degree of their compaction during rolling. Considering the fact that the composition of many grades of flux-cored strips, as a rule, includes from 4 to 12 and more components, differing by their composition, granulation and physico-mechanical properties, their sound mixing is an important factor, providing surfacing material of the required composition and properties.

The objective of the process of charge material mixing is making a product with as homogeneous as possible distribution of particles of the charge individual components and, therefore, possibly also more similar properties in any part of the produced mixture.

Two-cone mixers are the most often used for charge material mixing, which have a number of advantages, namely:

- they practically do not change the shape and dimensions of mixed material particles;

- ensure complete sealing;

- are readily cleaned, etc.

As shown by numerous studies, rotating mixers, operating in the optimum mode, are characterized by rather short mixing periods. If satisfactory blending of the charge is not achieved within 5 to $20 \mathrm{~min}$, further mixing is not rational and the cause preventing mixture blending should be sought. D. Kaufman [2] believes that 50 to 250 revolutions of the mixer (tentatively 10 to $25 \mathrm{~min}$ ) are enough to produce an equilibrium mixture, and $\mathrm{M}$. Ashton and F. Valentin [3] believe that the optimum period of mixture blending is within 1.2 to $18 \mathrm{~min}$.

By an established tradition, the time for blending the charge materials for flux-cored wires and strips is equal to $40-60 \mathrm{~min}$.

Influence of the time of charge material mixing on homogeneity of the charge for surfacing flux-cored strip PL-Np-500Kh40N40S2GRTs was studied. Experiments on charge mixing were conducted in a two-cone laboratory mixer of $7 \mathrm{dm}^{3}$ volume (charge mass of $3.35 \mathrm{~kg}$ ); two-cone industrial mixer of $150 \mathrm{dm}^{3}$ volume (charge mass of $100 \mathrm{~kg}$ ); two-cone industrial mixer of $600 \mathrm{dm}^{3}$ volume (charge mass of $419.42 \mathrm{~kg}$ ). Time of mix- 
Sampling time at mixing in mixers of different volume

\begin{tabular}{||c|c|c|c||}
\hline \multirow{2}{*}{ Batch number } & \multicolumn{3}{|c|}{ Mixing time, min, at mixer volume, $\mathrm{dm}^{3}$} \\
\cline { 2 - 4 } & 7 & 150 & 600 \\
\hline 1 & 5 & 5 & 5 \\
\hline 2 & 10 & 10 & 10 \\
\hline 3 & 15 & 15 & 15 \\
\hline 4 & 20 & 20 & 20 \\
\hline 5 & 30 & 30 & 30 \\
\hline 6 & 60 & 60 & 45 \\
\hline 7 & 120 & 120 & 60 \\
\hline 8 & 180 & 180 & 90 \\
\hline 9 & 240 & 240 & 120 \\
\hline 10 & - & - & 150 \\
\hline
\end{tabular}

ing and taking samples of individual charge batches are given in the Table.

24 samples for assessment of mixing quality were taken from each charge batch after mixing, by the method of quartering (at checking $7 \mathrm{dm}^{3}$ capacity mixer) or with a probe from different locations (at checking industrial mixers of 150 and $600 \mathrm{dm}^{3}$ volume). Two tablets were pressed from each odd sample. Sample analysis was performed in the ORTEC X-ray fluorescent analyzer Tefa.

Degree of charge component blending was evaluated by mean-root-square deviations of intensities by four elements: aluminium, silicon, chromium and iron. Obtained results are presented in Figure 1.

Analyzing the obtained results, a certain cyclicity of variation of mean-root-square deviations of intensities by all the elements, can be noted. Apparently, the phenomena of rather uniform blending of the charge composition alternate with segregation phenomena during manyhour processing in the mixer. The optimum mixing time, depending on mixer volume, is given in Figure 2.

The smallest mean-root-square deviation of intensities by most of the elements is observed in the mixer of $7 \mathrm{dm}^{3}$ volume after $10 \mathrm{~min}$ of mixing, in the mixer of $150 \mathrm{dm}^{3}$ - after 13 to $20 \mathrm{~min}$ of mixing, and in the mixer of $600 \mathrm{dm}^{3}-$ after $30 \mathrm{~min}$ of mixing that fully confirms the correctness of recommendations and data in published sources. These data show the principal possibility
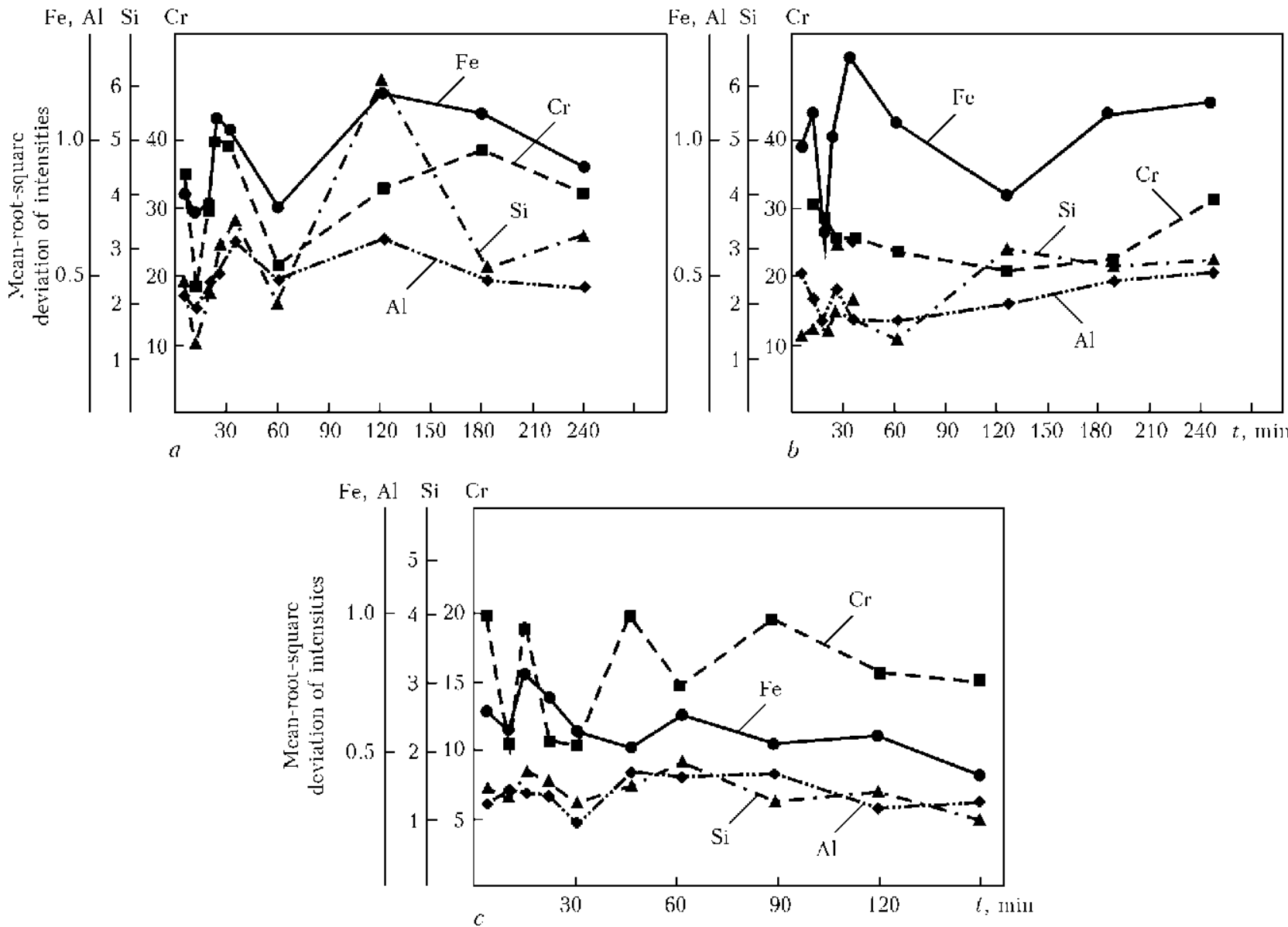

Figure 1. Influence of time $t$ of component mixing on mean-root-square deviation of intensities by individual elements: $a-$ for $7 \mathrm{dm}^{3}$ mixer; $b-150 \mathrm{dm}^{3} ; c-600 \mathrm{dm}^{3}$ 


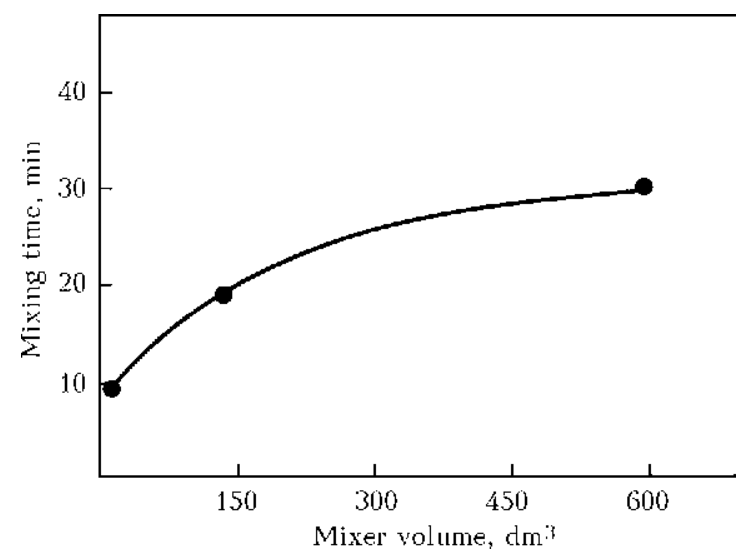

Figure 2. Optimum mixing time depending on mixer volume

of an abrupt shortening of operating time of twocone mixers that will greatly improve their efficiency.

It should be also noted that the composition of flux-cored strip or wire charge can be averaged not only due to their mechanical mixing, but also at application of complex-alloyed powders. The process of producing such powders was developed at PWI. Ingots of the specified composition are first melted from a mixture of ferroalloys and other alloying components with their subsequent plasma-arc thermocentrifugal atomization in an inert medium [4]. Obtained powder has spherically-shaped granules of the same composition, that essentially simplifies the process of surfacing material preparation, improves their weldingtechnological properties, as well as promotes an increase of deposited metal performance through reduction of its inhomogeneity.

The quality of flux-cored strip and its welding-technological properties, respectively, essentially depend on the degree of charge compaction during strip manufacture. Practical experience shows that insufficient compaction of the charge or its excessive compaction lead to lowering of welding-technological properties of flux-cored strip, in particular, to significant increase of spattering losses.

At present there is no objective characteristic of the quality of filler-powder compaction at flux-cored strip manufacture, and this parameter is totally dependent on operator experience.

Attempts at application of hydraulic load cell, built into the withdrawal-roll set of OB-2121 and OB-2240 mills, were unsuccessful. This engineering solution did not allow measuring the press-down force with the required accuracy, and also demonstrated the low reliability at operation under production conditions.

A new design of withdrawal-roll set was developed, which allows conducting continuous monitoring of withdrawal roll press-down force

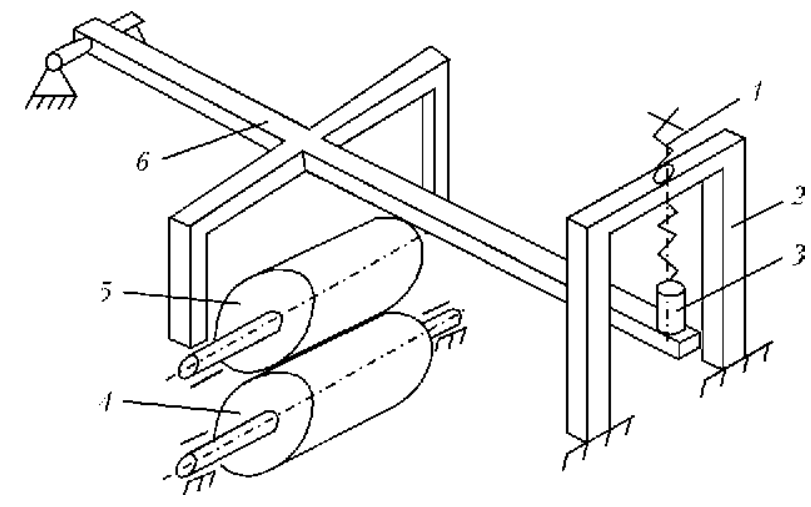

Figure 3. Kinematic scheme of withdrawal-roll set: 1 screw; 2 - stationary support; 3 - pressure sensor; 4 stationary roll; 5 - upper moving roll; 6 - lever; 7 axle

during flux-cored strip manufacture, as well as determining the optimum degree of filler-powder compaction (Figure 3). Press-down force is adjusted by screw 1 and is controlled by sensor 3, and the result is displayed on digital electronic indicator panel. The lever is designed so that the pressure is transmitted in two points to both the races of upper roll bearing through adjusting screws. This allows eliminating the roll non-parallelism, and, therefore, preventing flux-cored strip camber.

Influence of the degree of compaction on welding-technological properties of PL-Np400Kh20B7 M7B2F (PL-AN179) flux-cored strip was studied. Charge from one batch of stable composition was used to produce all the test fluxcored strips. Flux-cored strip filling coefficient was also constant. During flux-cored strip production just the withdrawal roll press-down force was varied. All the other parameters of mill adjustment remained unchanged, including fluxcored strip drawing speed.

At flux-cored strip production the withdrawal roll press-down force was set within the follow-

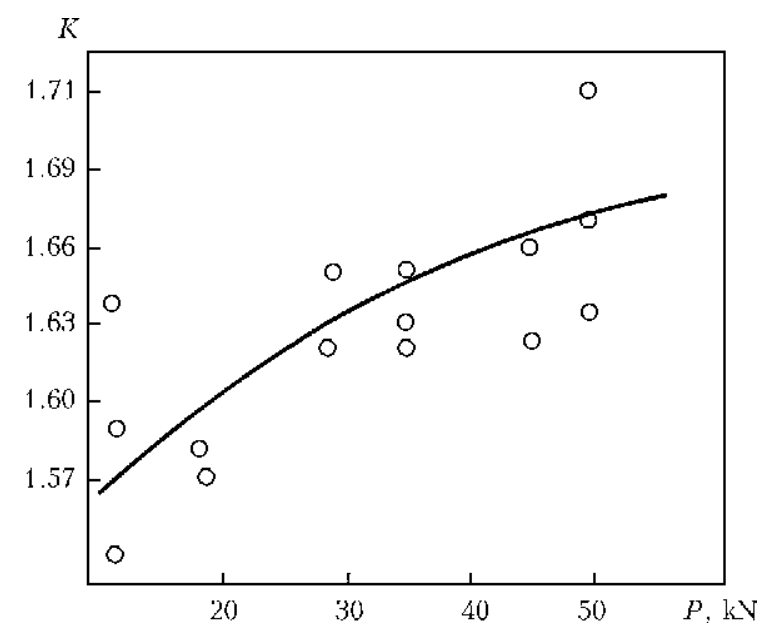

Figure 4. Degree of filler-powder compaction 


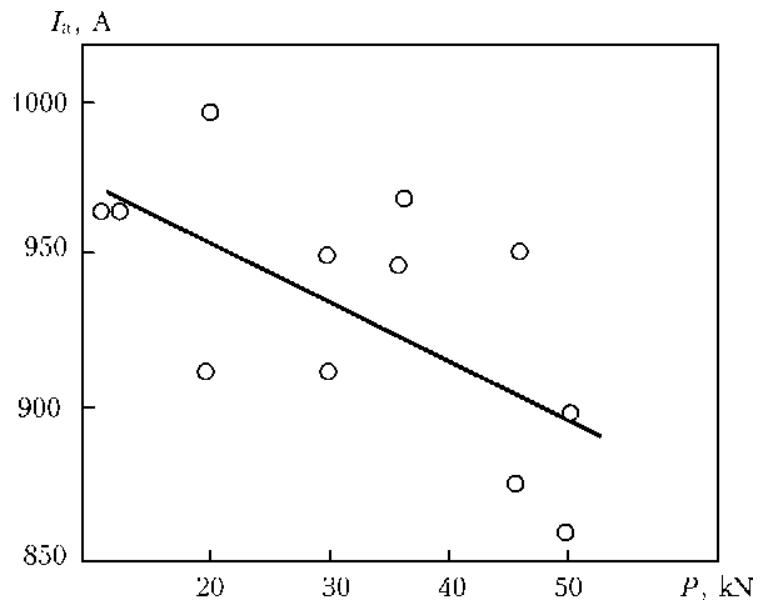

Figure 5. Surfacing current depending on roll press-down force

ing ranges, $\mathrm{kN}: 2.5-3.5 ; 15-20 ; 36-40 ; 48-53$; $66-72 ; 75-81$.

Degree of charge compaction in flux-cored strips was determined, depending on withdrawal roll press-down force. It was assessed as the ratio of apparent density of filler-powder after its mixing to density of filler-powder in the flux-cored strip by the following formula (Figure 4):

$$
K=\rho_{1} / \rho_{2},
$$

where $\rho_{1}$ is the apparent density of the charge after component mixing; $\rho_{2}$ is the charge density in the flux-cored strip.

Degree of charge compaction increases proportionally to increase of roll press-down force, and at press-down force of $60 \mathrm{kN}$ it is equal to 1.66. When studying the electrode material welding-technological properties, surfacing with test flux-cored strips was performed in A-874N apparatus with DC power source VDU-1201. Surfacing modes were as follows: voltage of $29 \pm 1 \mathrm{~V}$; flux-cored strip feed rate of $41 \pm 1 \mathrm{~m} / \mathrm{h}$; surfacing

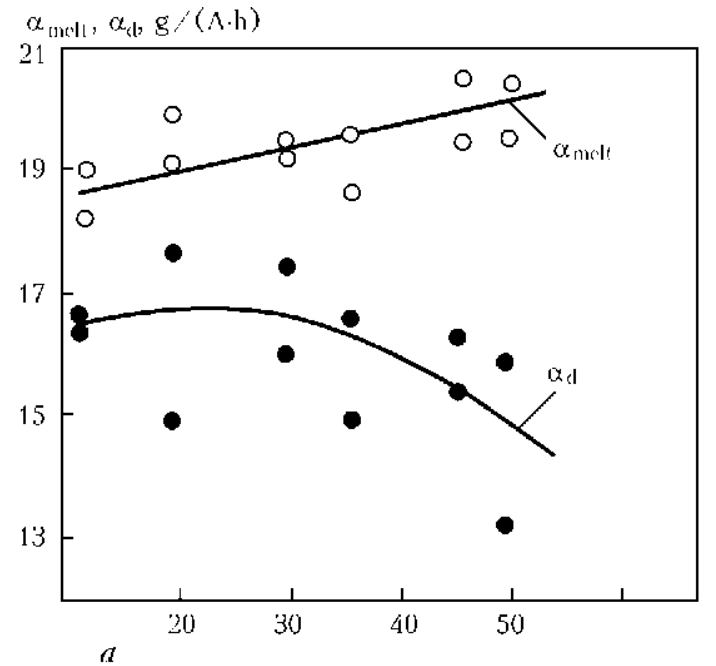

speed of $19 \pm 1 \mathrm{~m} / \mathrm{h}$; electrode extension of $50 \pm$ $\pm 5 \mathrm{~mm}$; at reverse polarity.

Surfacing was performed on St3 steel plates of $20 \times 45 \times 300 \mathrm{~mm}$ size. During surfacing by each of test flux-cored strips average current and voltage were recorded, and coefficients of current and voltage variation were determined. ANP-2 information-measurement system based on «Elektronika-60M» microcomputer was used to obtain these data.

Coefficients of melting, deposition and metal losses for evaporation and spattering were determined by the following dependencies.

Melting coefficient $\alpha_{\text {melt }}$ :

$$
\alpha_{\text {melt }}=\frac{3600\left(m_{\mathrm{str}}-m_{\mathrm{str}}^{\prime}\right)}{I t},
$$

where $m_{\text {str }}, m_{\text {str }}^{\prime}$ are the strip mass before and after surfacing, respectively; $I$ is the surfacing current, $\mathrm{A} ; t$ is the surfacing time, s.

Deposition coefficient $\alpha_{d}$ :

$$
\alpha_{\mathrm{d}}=\frac{3600\left(m_{\mathrm{pl}}-m_{\mathrm{pl}}^{\prime}\right)}{I t}
$$

where $m_{\mathrm{pl}}, m_{\mathrm{pl}}^{\prime}$ is the plate mass before and after surfacing, $g$.

Coefficient of losses for evaporation and spattering $K_{1}$ :

$$
K_{1}=\frac{\left(m_{\mathrm{str}}-m_{\mathrm{str}}^{\prime}\right)-\left(m_{\mathrm{pl}}-m_{\mathrm{pl}}^{\prime}\right)}{m_{\mathrm{str}}-m_{\mathrm{str}}^{\prime}} .
$$

Losses for spattering:

$$
K_{\mathrm{sp}}=\frac{m_{\mathrm{sp}}}{m_{\mathrm{str}}-m_{\mathrm{str}}^{\prime}},
$$

where $m_{\mathrm{sp}}$ is the collected spatter mass.

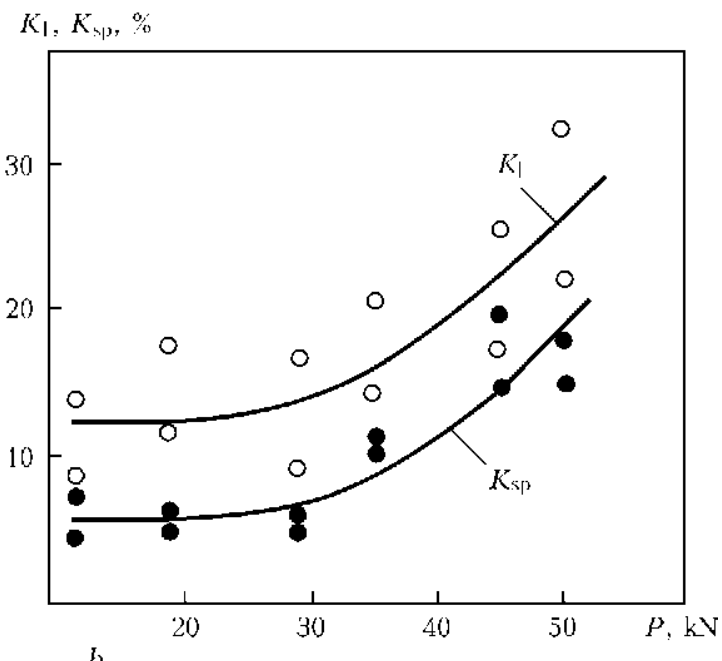

Figure 6. Dependence of coefficients of melting and surfacing $(a)$ and coefficients of losses and spattering (b) on roll press-down force 

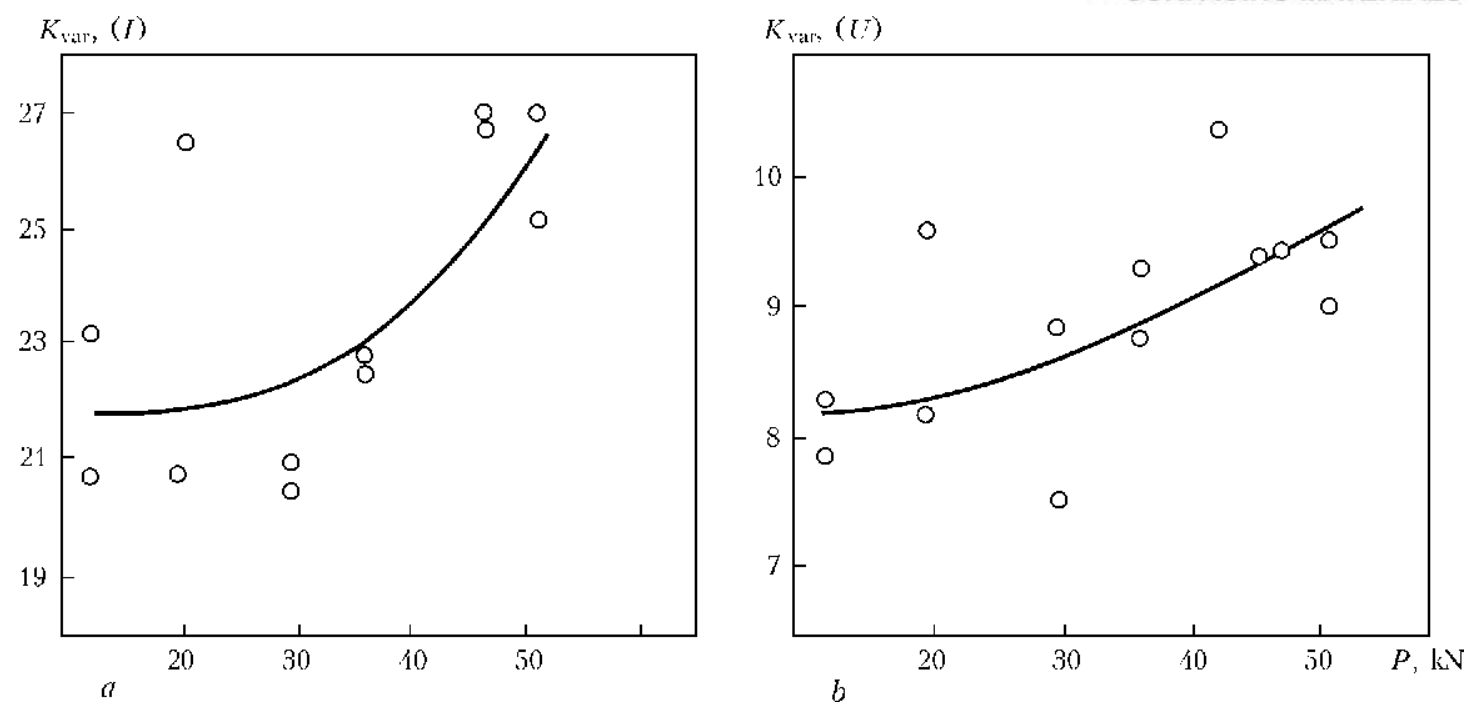

Figure 7. Coefficient of variation of current $(a)$ and voltage $(b)$ depending on roll press-down force

With increase of the degree of charge compaction, current decreases at unchanged rate of fluxcored strip feed, i.e. at the same arc voltage, the arc power consumed for melting of one and the same quantity of electrode material, becomes smaller (Figure 5).

This leads to increase of melting coefficient, but deposition coefficient decreases at roll press-down forces above $40 \mathrm{kN}$ (Figure 6, $a$ ). Decrease of deposition coefficient is attributable to an abrupt increase of spattering losses (Figure 6, $b$ ).

Increase of the coefficients of variation of current and voltage is also observed in the same range (press-down force from 40 to $80 \mathrm{kN}$ ) ( $\mathrm{Fi}$ gure $7, a, b)$.

Thus, increase of the degree of compaction of flux-cored strip charge leads to increase of electrode material melting rate, but this positive effect is completely neutralized by an abrupt increase of spattering losses.

Optimum welding-technological properties of flux-cored strip in the considered case were obtained only at roll press-down force in the range of 20 to $40 \mathrm{kN}$. At press-down forces below $20 \mathrm{kN}$, the result repeatability is low, that is, obviously, related to insufficient degree of flux-cored strip charge compaction.

Increase of electrode material melting rate is attributable to the change of electric resistance of flux-cored strip, that is, apparently, associated with the change of strip-sheath resistance due to work hardening and change of the cross-section at its deformation, because of imprints of withdrawal rolls of specified configuration on its surface.

Increase of spattering with increase of the degree of charge compaction, in our opinion, is associated with the change of charge heat conductivity and lowering of its electric resistance.

Conducted studies were the basis for improvement of technological manual for manufacturing of this grade of flux-cored strip, that allowed stabilization of manufactured product quality and reducing its losses at surfacing.

Application of the new technology of fluxcored strip manufacture allowed an essential reduction of equipment overall dimensions and several times lowering the electrode material power consumption.

\section{Conclusions}

1. It is established that the optimum time of mixing the components of flux-cored strip core charge depends on mixer volume and varies in the range of 10 to $30 \mathrm{~min}$. Increase of mixing time above these limits lowers the charge homogeneity.

2. A system was developed for determination of press-down force of rolls in withdrawal-roll set of flux-cored strip manufacturing mill. Optimum ranges of compaction of flux-cored strip charge were determined. Insufficient degree of compaction, or its increased value adversely affect the welding-technological properties of electrode material. This leads to greater spattering and lower stability of flux-cored strip melting.

3 . Obtained investigation results were implemented in the technology and equipment for manufacturing flux-cored strips.

1. Zhudra, A.P., Voronchuk, A.P. (2012) Cladding flux-cored strips (Review). The Paton Welding J., 1, 34-38.

2. Kaufman, J.J. et al. Ultrasonic bone-therapy apparatus and method. Pat. 5547459 U.S. Publ. Aug. 20, 1996.

3. Ashton, M.D., Valentin, F.H. (1986) The mixing of powders and particles in industrial mixers. Transact. of Inst. Chem. Engrs., 44(5), 166-169.

4. Zhudra, A.P., Krivchikov, S.Yu., Dzykovich, V.I. (2014) Application of complex-alloyed powders produced by thermocentrifugal sputtering in flux-cored wires. The Paton Welding J., 12, 36-40. 